\title{
Mechanical Bending and Modulation of Photoactuation Properties in a One-Dimensional $\mathrm{Pb}(\mathrm{II})$ Coordination Polymer
}

\author{
Bibhuti Bhusan Rath, ${ }^{1}$ and Jagadese J. Vittal, ${ }^{1, *}$ \\ ${ }^{1}$ Department of Chemistry, National University of Singapore, Singapore 117543 \\ Key words: Coordination Polymer $\bullet$ Flexible crystal $\bullet$ Photoactuation $\bullet$ Dual stimuli responsive
}

\begin{abstract}
With emergent research on stimuli responsive materials, dynamic crystals are at the forefront of investigation. However, research on the mechanical properties of coordination polymers (CPs) is still in its infancy. Elastic deformation induced by pressure and photoactuation are rare occurrences in CPs, let alone their combination in a single CP. Here, we report a one-dimensional (1D) CP comprising $\mathrm{PbBr}_{2}$ chains with 3-fluoro-4'-styrylpyridine arms showing excellent elasticity and photomechanical properties. A slender crystal can be bent to make a circle and write different shapes with restoration of original shape upon removal of the applied force. In addition, photomechanical properties triggered by [2+2] cycloaddition of the olefinic ligand can be modulated easily by variation of the crystal sizes. Crystals with bigger width show destructive photosalient effects while the smaller ones show plastic deformation like bending, twisting, curling etc. upon UV irradiation. This example provides avenue for designing CPs for multi-stimuli responsive actuating properties.
\end{abstract}

\section{INTRODUCTION}

In recent years, great efforts have been made to mimic nature artificially for fabricating actuators that can work with accuracy in response to external stimuli by transduction of energy. ${ }^{1-4}$ Mechanically responsive actuators hold tremendous potential for their use in smart medical devices, artificial muscles, flexible electronics etc. ${ }^{1,}$ 5-7 A new class of macroscopic crystals also known as "soft crystals" exhibit mechanical movements induced by pressure, heat, and light which often results in hopping, splitting, bending, curling, coiling, twisting, swimming of the crystals. 1 8-26 With the upsurge of dynamic crystals, several examples of mechanically flexible crystals have been reported in the literature showing elastic and plastic deformation upon the application of an external force. ${ }^{27-34}$ Apart from actuation by mechanical force, photomechanical actuation is of great advantage due to its remotely controlled process. ${ }^{25}$, 35-37 Examples of flexible crystals and photomechanical actuators have been reported separately in the literature where most of them are organic crystals. ${ }^{1,2}$, 5, 27 However, multi-stimuli responsive crystals are rarely encountered in the literature. $1,8,13,38$ In general, fabrication of multifunctional materials demands the combination of several functionalities for imbibing multiple properties. Designing single component smart materials that can perform multiple functions in response to different stimuli is a challenge in fundamental chemistry as well as materials fabrications.

Apart from their catalytic, magnetic, and optical properties, coordination polymers (CPs) have recently been explored for their mechanical flexibility and photoactuation properties. ${ }^{35}, 38,39$ Elasticity $^{38,} 39$ and plasticity ${ }^{40}$ have been investigated in one-dimensional (1D) CPs having similar structures. Similarly, photoactuating CPs showing photosalient effect ${ }^{35}, 41$ and photomechanical motions ${ }^{42}$ have been reported in separate literature articles. However, CPs showing multi-stimuli responsive behavior are rarely observed. ${ }^{4,38}$ Most importantly, modulation of the actuation properties in a single crystalline material is a great challenge and it has been achieved only in organic crystals. 43 , 44 Controlled modulation of mechanical responsiveness in CPs can pave their way to emerging applications in wearable technology and tissue engineering etc. Multistimuli receptive actuators based on CPs can outshine organic counterparts due to their additional properties originating from the metal centers.

In this paper we report a $1 \mathrm{D} \mathrm{CP}$ of $\mathrm{Pb}(\mathrm{II}),\left[\mathrm{PbBr}_{2}(3 \mathrm{~F}-\right.$ spy) $)_{2}$ ( $3 \mathrm{~F}$-spy $=3$-fluoro-4'-styrylpyridine) which not only shows great mechanical flexibility but also modulated photoactuation properties. The presence of anisotropic weak interactions along the crystal lattice facilitates the restoration of crystal deformation upon removal of external force, enabling slender crystals to show mechanical elasticity. In addition, macroscopic crystals show a wide range of photoactuating properties depending on their sizes. Violent photosalient effects such as jumping, breaking, splitting effects as well as bending, twisting, curling etc. have been observed in the 1D CP. To the best of our knowledge, we are not aware of any CP showing such multi-actuation properties.

\section{RESULTS AND DISCUSSION}

Structural Analysis. The 1D CP $\left[\mathrm{PbBr}_{2}(3 \mathrm{~F}-\mathrm{spy})_{2}\right]$ 1, was synthesized as single crystals by slowly evaporating a DMF solution containing 1:2 molar ratio of $\mathrm{PbBr}_{2}$ and 3F-spy. Single crystal structure at 295(2) K reveals that 1 
crystallized in the monoclinic space group $P 2{ }_{1} / c$ with $\mathrm{Z}=2$. The asymmetric unit constitutes half the formula of the compound. The $\mathrm{Pb}(\mathrm{II})$ atom occupies the crystallographic center of inversion and has octahedral geometry from two 3F-spy ligands coordinating via $\mathrm{N}$ atoms and four bridging bromide ions. The 3F-spy ligands are flanked in trans positions. The bridging bromide ions bind neighbouring $\mathrm{Pb}$ (II) atoms forming $\mathrm{Pb}(\mu-\mathrm{Br})_{2}$ chains which propagate along the $a$-axis. All the $3 \mathrm{~F}$-spy ligands are arranged parallel in their respective side of the $\mathrm{Pb}(\mu-\mathrm{Br})_{2}$ chain in a head-tohead manner (Figure 1, Figure S3-S5). The distance between the centers of the olefin bonds of the styryl groups is $4.175 \AA$ ( $a$-axis length), which falls in the upper limit of the required distance $\left(\begin{array}{ll}4.2 & \AA\end{array}\right)$ satisfying Schmidt's topochemical criteria ${ }^{45}$ for the [2+2] cycloaddition reaction in the solid state upon UV irradiation. ${ }^{46,47}$ This could lead to the formation of another 1D dimerized structure upon UV light irradiation.

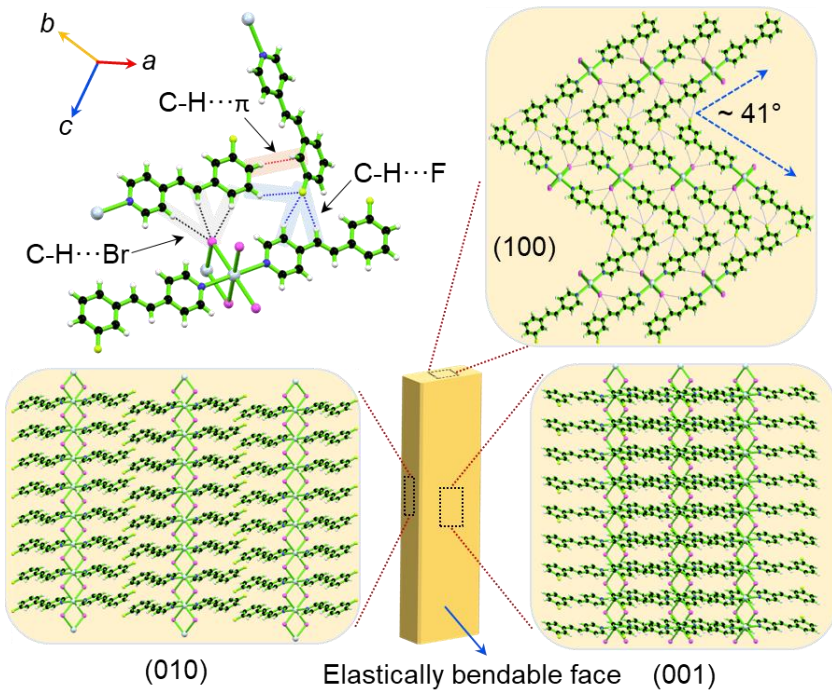

Figure 1. Weak interactions between neighboring groups and crystal packing as seen perpendicular to the crystal faces.

Elastic Bending. Elastic ${ }^{39}$ and plastic ${ }^{40}$ bending have been reported in $1 \mathrm{D}$ CPs having similar ribbon like structures. Elasticity is observed when "strong" interchain interactions prohibit the formation of stable interwoven networks upon the application of mechanical force. ${ }^{39,40} \mathrm{We}$ tested this interesting elastic deformation on slender crystals of $\mathbf{1}$ as it also possesses the key structural prerequisite. ${ }^{39}$ After fixing one end of the crystal, with the help of a metal needle the other end of the crystal was bent along the crystal length, i.e., by applying the force perpendicular to the 1D chains. When the force was applied on the (010) plane, crystals broke immediately, while flexible bending was observed when poked on the (001) face. Macroscopic elastic crystal deformation occurred to a great extent without any fracture and the bent crystal underwent transient recovery upon withdrawal of the external force. This could be repeated for several times in both the directions indicating great flexibility of the crystals (Figure 2a, Video SV1). With the help of Paratone oil we tried to bend the crystals to such an extent that we could write a few letters with them (Figure S7). Interestingly, a full circle could be made with a typical slender crystal (4 $\mathrm{mm} \times 23 \mu \mathrm{m} \times 15 \mu \mathrm{m}$ ) indicating excellent flexibility of the
CP. A crystal of $\sim 4 \mathrm{~mm}$ length could be flexed into a loop of $\sim 0.5 \mathrm{~mm}$ radius (circumference $3.14 \mathrm{~mm}$ ) (Figure S8, Video SV2). Further, a "critical radius" of $\sim 0.2 \mathrm{~mm}$ was attained before the crystal broke (Video SV2). Elastic bending strain $\varepsilon=h / 2 \mathrm{R}$ (where $\mathrm{h}$ is the thickness of the crystal and $\mathrm{R}$ is the radius of the circle) was calculated for several crystals and the average bending strain was found out to be $\sim 1.3 \%$, which is higher than reported elastic CPs. ${ }^{39}$

Mechanism of Bending. Diffraction images were collected before and after bending experiments at several positions of the crystals. Sharp diffraction peaks with similar cell data prior to bending suggested that long range ordering in the crystal is restored without any substantial damage even after repeated bending experiments (Figure S10, Table S3). This indicates reversible reconfiguration of the local environment at the bent/curved positions due to supramolecular interactions (Figure 1). However, diffraction spots of a crystal under a tensile state could not be indexed. As per the previous reports, elastic deformation is a consequence of the gradient of stretching to compression from the outside to the inside of a bent crystal. ${ }^{28,32,38,39}$ Slight displacement of the molecules at the bent position results in the diffused diffraction pattern (Figure S10), unsuitable for indexing. However, consensus of this crystal being single is very clear as proven by previous examples. $^{7}$

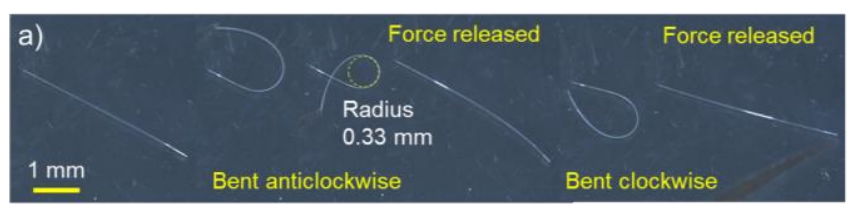

b)

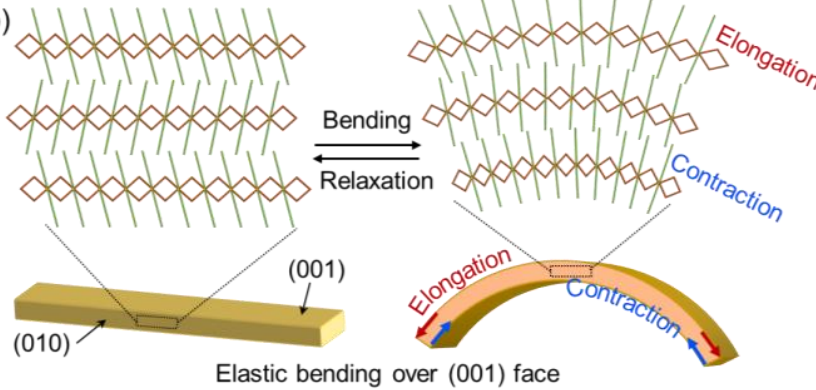

Figure 2. (a) Single crystal of $\mathbf{1}$ showing elastic bending upon application of an external force. (b) Plausible mechanism of elastic bending.

Elastic bending in 1D CPs of Cd(II) has been explained in terms of supramolecular interactions. ${ }^{39}$ Similarly, in our case, all 1D polymeric chains are interlinked together by $\mathrm{C}$ $\mathrm{H} \cdots \mathrm{F}$ and $\mathrm{C}-\mathrm{H} \cdots \mathrm{Br}(\mathrm{Pb})$ interactions perpendicular to the crystal elongation i.e., crystallographic $a$-axis (Figure 1 and Table S2). Due to the presence of such "strong" interactions between the neighboring chains, an interchain network is formed throughout the crystal. Thus, 1D chains are interlocked, preventing a slippage and further plastic deformation upon application of an external force. However, it is important to mention that both elastic ${ }^{28}$ and plastic ${ }^{40}$ bending have been reported in the absence of slip planes. Anisotropic distribution of supramolecular interactions in the crystal lattice facilitates mechanical flexibility in the macroscopic crystals, as observed in elastic crystals of copper(II) acetylacetonate complex ${ }^{28}$, and Cd- 
based $1 \mathrm{D} \mathrm{CPs}^{39}$. In addition, the $\mathrm{Pb}(\mu-\mathrm{Br})_{2}$ unit is highly tensile as seen from the data collected at 100(2) K (Figure S4). Similar structural motif in a CP showed temperature dependent reversible flexibility. ${ }^{23}$ So the application of an external force compel the units to stretch on the outer arc and compress on the inner arc of a bent crystal, thus constraining the ligands to accommodate accordingly (Figure $2 \mathrm{~b}$ ). The neighbouring $1 \mathrm{D}$ chains are slanted at $\sim 41^{\circ}$ to each other and can adjust reversibly as seen from the packing perpendicular to (010) plane (Figure 1). Hence, elastic bending is only feasible over the (001) plane and the pristine structure is reinstated upon removal of the mechanical force (Figure 2b). Apart from the structural interactions, thickness of the (010) plane was found to be an important parameter for the mechanical bendability of the crystals.

Photoreactivity. Aimed at achieving a multifunctional $\mathrm{CP}$, the inclusion of a photoreactive olefinic ligand was deliberate. As per the structural arrangement in the 1D CP, the styryl groups of 3F-spy ligands are expected to undergo [2+2] dimerization upon UV irradiation to form cyclobutane rings. When single crystals of different dimensions were irradiated under UV light (360 nm) using MAX-350 with a $300 \mathrm{~W}$ xenon light source, various photomechanical effects of the crystals were observed, which subsided within few minutes. Crystal residues were dissolved in DMSO- $d_{6}$ for ${ }^{1} \mathrm{H}$ NMR spectral analysis. Integration of the peaks revealed that the signal of pyridyl protons of 3F-spy at $8.57 \mathrm{ppm}$ decreased and new peaks at $8.34 \mathrm{ppm}$ and $4.61 \mathrm{ppm}$ evolved, which are ascribed to pyridyl protons and cyclobutane protons of rctt-3F-ppcb (rctt-3F-ppcb = rctt1,2-bis(4'-pyridyl)-3,4-bis(3'-fluoro-phenyl)cyclobutane) respectively. The photoconversion of $3 \mathrm{~F}$-spy to rctt-3Fppcb was calculated to be $49 \%$ indicating [2+2] cycloaddition reaction was the driving force for the photomechanical effects. Complete photodimerization of the ligands occurred after 15 min of UV irradiation resulting in the formation of a new 1D CP 2 (Figure 3).

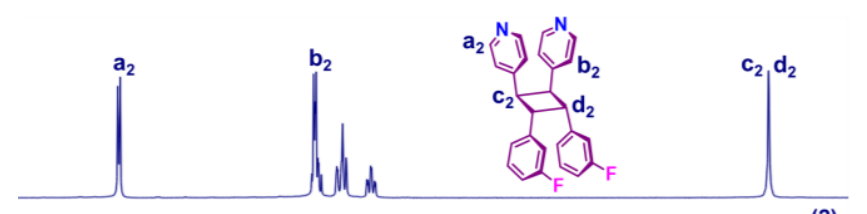

(2)

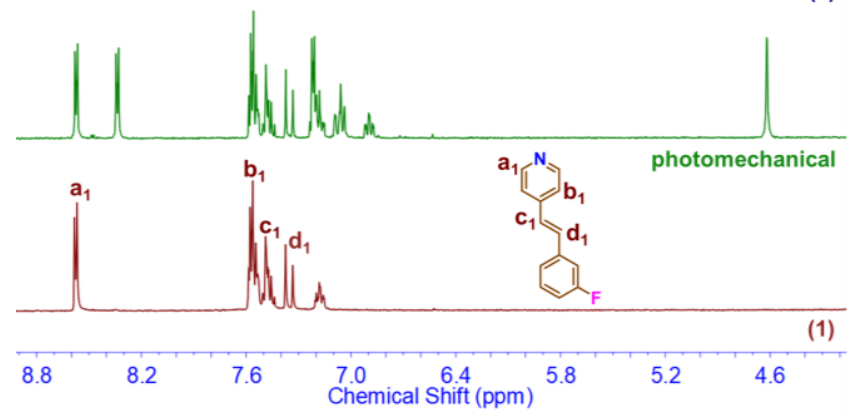

Figure 3. ${ }^{1} \mathrm{H}$ NMR spectra of $\mathbf{1}, \mathbf{1}$ after showing photomechanical effects and the dimerized CP 2. Relevant proton peaks are assigned.

In our previous work, we could obtain the single crystal structures of partial photoconverted and 100\% dimerized product of a photosalient CP. ${ }^{35}$ In this case, such attempts were unfruitful as the crystals could not be indexed due to deterioration of the single-crystal quality. The PXRD pattern of $\mathbf{2}$ closely matches with that of $\mathbf{1}$ with peaks shifting to the lower angles, which suggests a lattice expansion after the photoconversion (Figure S1). Our attempts to solve the structure from the powder data is not successful.

Modulation of Photoactuation. After our successful attempt at understanding the photosalient effect and photomechanical motions in a $1 \mathrm{D} \mathrm{CP}^{35}$, here we try to modulate the photoactuation properties in different dimensions of slender crystals. Naumov et al. have explained various photomechanical effects of crystals in terms of several factors including crystal size/shape and surface strain tensor. ${ }^{1}, 5$ Modulation of photoactuation properties have been achieved in some organic crystals, 43,44 but controlled modulation of such properties in CPs is not reported. In order to rationalize and successfully modulate different photomechanical motility, we grew crystals of several distinguished dimensions. Instead of pure DMF as the crystallization solvent, different ratios of DMF and $\mathrm{MeCN}$ resulted in the formation of long single crystals of different width and thickness. We were able to grow at least four different crystal dimensions and investigated their photomechanical behavior in detail under a microscope equipped with a high-resolution camera.

Crystals with width $\times$ thickness larger than $85 \mu \mathrm{m} \times 45$ $\mu \mathrm{m}$ mostly showed violent photosalient effects, exhibiting jumping and splitting of crystals during $90 \mathrm{~s} \mathrm{UV}$ irradiation (Figure 4a-c, Video SV3). Initially bending along the crystal length occurred when the crystals were irradiated perpendicular to the (001) face. Crystals with smaller width started splitting and breaking after $20 \mathrm{~s}$, while bigger ones took $5 \mathrm{~s}$ more. In general, continuous stress relaxation in the absence of an induction period results in bending while rapid stress relaxation after an induction period leads to breaking, jumping of the crystals. ${ }^{5}$ Prolonged irradiation resulted in explosion of all the crystals into fragments and the fragmented crystals jumped to move away from the visual field of the microscope with few portions left on the glass slide (Figure 4a, Video SV3). Most fragmentation occurred along the (100) plane following the bending along the longest crystallographic face i.e., (001) plane. Scattered fragments showed only $\sim 29 \%$ photodimerization after the end of violent PS effects. Similar observations have been made in other photosalient crystals, where percentage conversion is small for the observed effects. ${ }^{20,} 48$ This is possibly related to a sudden anisotropic expansion in the unit cell during dimerization or heterogeneous product distribution in the crystal as explained in our prior work..$^{35}$ Formation of cyclobutane rings between neighbouring $3 \mathrm{~F}$ spy ligands cause high strain in $\mathrm{Pb}(\mu-\mathrm{Br})_{2}$ units resulting the disruption of $\mathrm{PbBr}_{2}$ chains that propagate along the $a$-axis.

In general, bending was observed in crystals of width $\times$ thickness below $65 \mu \mathrm{m} \times 25 \mu \mathrm{m}$. Elaborate photobending experiments were conducted by irradiating single crystals glued to a glass fiber. Irradiation on (001) or (001) plane resulted in bending of the crystal away from the light source (Figure 4d, Video SV4). Initial irradiation results in the formation of a product phase on the irradiated surface, thus generating a strain gradient between two faces. Flexibility of $\mathrm{PbBr}_{2}$ chains accommmodate the strain by facilitating bending along the crystal length. A slender crystal $(3.2 \mathrm{~mm}$ $\times 31 \mu \mathrm{m} \times 16 \mu \mathrm{m}$ ) would bend to a great extent forming a 

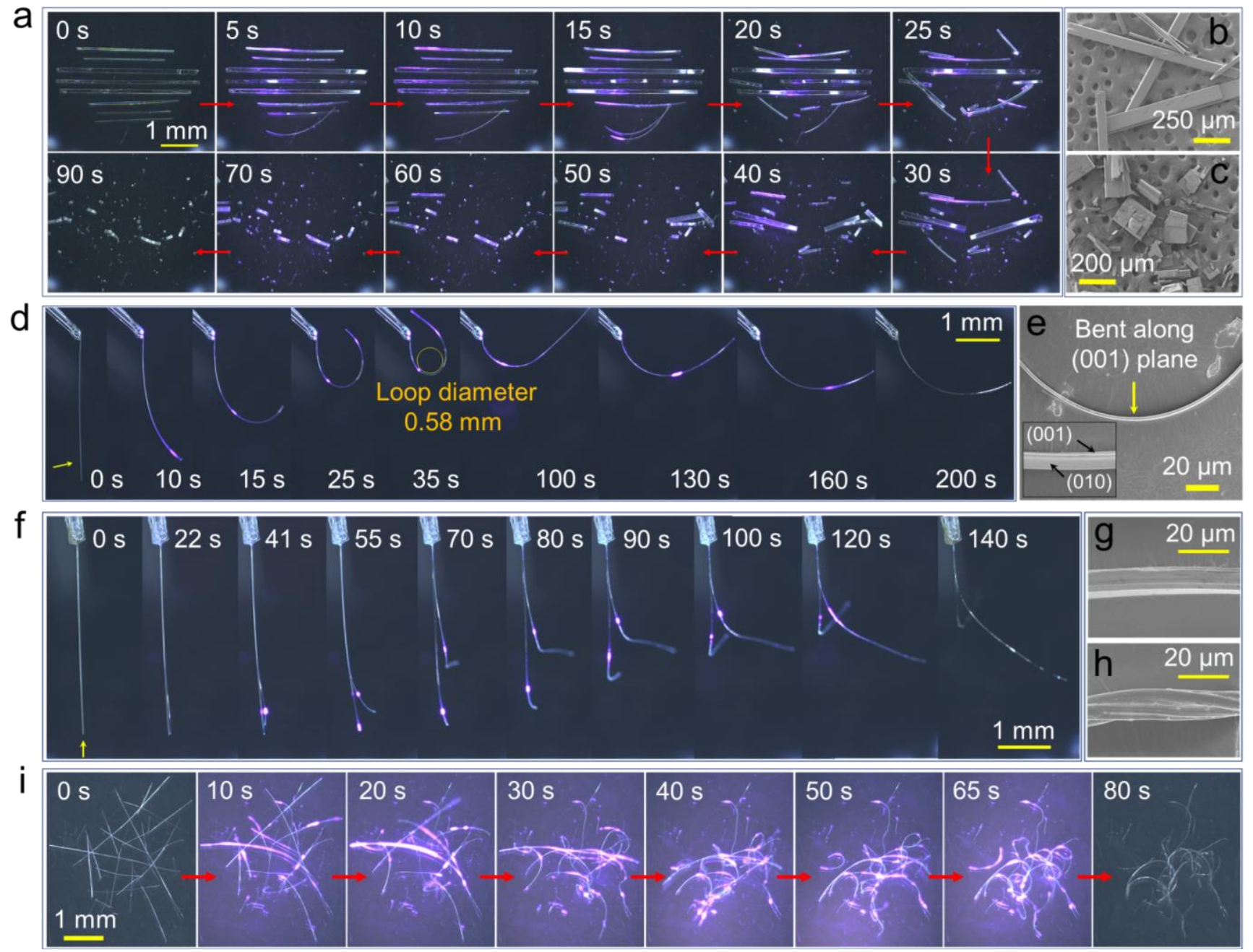

Figure 4. (a) Photosalient effect in thicker crystals of 1. SEM images of crystals (b) before (c) after PS effect. (d) Photoinduced crystal bending. (e) SEM image of a bent crystal after UV irradiation. (f) Photoinduced crystal splitting and twisting. SEM images of crystal (g) before and (h) after photoinduced twisting. (i) photoinduced curling of fiber crystals.

loop (diamter $0.58 \mathrm{~mm}$ ) after $30 \mathrm{~s}$. However, upon continued UV exposure the crystal undergoes a slow release of internal strain by bending backwards (Figure 4d, Video SV4). Although it seems like an elastic motion, the crystal undergoes a plastic deformation due to [2+2] photocycloaddition. SEM image of a bent crystal after UV irradiation clearly shows plastic bending over the (001) face (Figure 4e). Beacuse of higher flexibility, longer crystals ( $>3 \mathrm{~mm}$ ) tend to bend backwords after completing a loop while relatively shorter ones $(<2 \mathrm{~mm})$ just moved away from the light source to a bent position. In general, irradiation on the upper half (free end) of the crystal generated faster response and the movement was minimal when the other end sticking to the glass rod was exposed. Also, when the crystals underwent bending away from the UV light, it was turned off at the middle and the irradiation direction was reversed to bend the crystal back to its original position (Figure S11, Video SV5).
Splitting of crystal along its length was observed when it was irradiated by a pointed beam from the UV light source at the tip i.e., (100) plane (Figure 4f). It took more than 20 seconds for any observable effect possibly due to the exposure of smaller surface area to UV light. Dimerization on the (100) face created strain in the parallel 1D chains slanted $\sim 41^{\circ}$ to each other, and the crystal split along the longest crystal dimension i.e., parallel to (001) face to release the surface strain. Further, bending and twisting of the split portions were observed during $140 \mathrm{~s}$ irradiation time (Figure 4f, Video SV6). Hence the position of the UV irradiation is an important aspect for the induced photomechanical effects. Apart from bending, twisting of the crystal is fascinating. When free-standing crystals were irradiated over glass slide, pronounced jumping, and bending happened, while crystals were also seen to twist. $60 \mathrm{~s}$ UV irradiation resulted plastically deformed bent and twisted crystals (Figure S12, Video SV7). SEM images of pristine crystal and photoinduced twisted crystal highlight the change (Figure 4g-h). 
Further, reducing crystal width $\times$ thickness below $18 \mu \mathrm{m}$ $\times 10 \mu \mathrm{m}$ resulted in long fiber crystals. Photoinduced curling was peculiar in such crystals. Crystals underwent bending along the length from one end or both the ends to form plastically curled crystals (Figure 4i, Video SV8). Crystals interweaved with each other and the conglomerate looked like curly hair. Generally, crystals of smaller thickness showed better curling compared to relatively bigger ones. A bunch of fiber-like crystals placed over one another immediately curled and intertwined together upon UV irradiation to form tangled bunch of fibers (Figure S13, Video SV9). Curling of crystals have been studied in organic crystals ${ }^{17,43,49,50}$ and no such photomechanical behavior has been reported in CPs. Transduction of energy for performing observable work is of great importance for actuating materials. So, we tasked a bunch of slender crystals in performing mechanical work by moving a glass rod $~ 10$ times higher than their weight upon UV irradiation (Figure S14, Video SV10). This is a clear demonstration of the conversion of light energy to mechanical energy.

\section{CONCLUSION}

In summary, a 1D CP was found to be showing dual stimuli responsive actuation properties. Comparison of structural arrangements with previous examples of CPs assisted in rationalizing the elasticity of slender crystals. Presence of anisotropic supramolecular interactions facilitates crystal bending over (001) plane only. Diffused diffraction peaks at the bent position could not be indexed due to the stressed structure. However, a relaxed crystal retained the same cell parameters as that of a pristine one, confirming the reversible reconfiguration. In addition to this, our rational inclusion of an olefinic ligand in the $\mathrm{CP}$, enabled us to study photomechanical actuation properties triggered by [2+2] cycloaddition reaction. In general, the stress generated in the crystal during the photochemical reaction results in various photomechanical motions depending on the strain tensor and crystal thickness. Modulation of photoactuation properties has been manifested in organic crystals. Addition of metal component in the crystal has not resulted in such varied response under UV illumination. We could successfully modulate the photoactuation properties of the CP by varying crystal thickness. Violent photosalient effects were prevalent in wider crystals, while photobending, twisting, curling etc., were observed in slender crystals of smaller width. Crystal splitting could be seen in bendable crystals depending on the position of light irradiation. Such actuation properties have seldom been reported in CPs. This example can provide important new insights for rationally designing multi-actuator materials which can conveniently convert energy into mechanical work. Control and manipulation of actuation properties will provide avenue for accuracy and precision in such applications.

\section{ASSOCIATED CONTENT}

\section{Supporting Information.}

Experimental details, crystallographic data, PXRD, TGA, optical microscopic images (PDF)

Crystallographic data in cif format (CIF)

Video SV1: Elastic bending

Video SV2: Mechanical bending

Video SV3: Photosalient effects

Video SV4: Photoinduced bending

Video SV5: Light direction dependant bending

Video SV6: Photoinduced splitting and twisting

Video SV7: Photoinduced bending and twisting

Video SV8: Photoinduced curling

Video SV9: Photoinduced curling

Video SV10: Photoinduced work by crystals

\section{AUTHOR INFORMATION}

\section{Corresponding Author}

* Jagadese J. Vittal - Department of Chemistry, National University of Singapore, Singapore 117543 (ORCID: 0000-00018302-0733) email: jjvittal@u.nus.edu

\section{Author}

Bibhuti Bhusan Rath - Department of Chemistry, National University of Singapore, Singapore 117543 (ORCID: 0000-00025323-6936) email: bbrath@u.nus.edu

Notes

The authors declare no competing financial interest.

\section{ACKNOWLEDGMENT}

This work was financially supported by the Ministry of Education, Singapore (Grant No. Tier 1 R-143-000-B13-114). We acknowledge the help of Geok Kheng Tan for collecting the single crystal X-ray intensity data.

\section{REFERENCES}

1. Naumov, P.; Karothu, D. P.; Ahmed, E.; Catalano, L.; Commins, P.; Mahmoud Halabi, J.; Al-Handawi, M. B.; Li, L., The Rise of the Dynamic Crystals. J. Am. Chem. Soc. 2020, 142, 1325613272.

2. Y Yu, Q.; Aguila, B.; Gao, J.; Xu, P.; Chen, Q.; Yan, J.; Xing, D.; Chen, Y.; Cheng, P.; Zhang, Z.; Ma, S., Photomechanical Organic Crystals as Smart Materials for Advanced Applications. Chem. Euro J. 2019, 25, 5611-5622.

3. Dong, X.; Tong, F.; Hanson, K. M.; Al-Kaysi, R. O.; Kitagawa, D.; Kobatake, S.; Bardeen, C. J., Hybrid Organic-Inorganic Photon-Powered Actuators Based on Aligned Diarylethene Nanocrystals. Chem. Mater. 2019, 31, 1016-1022.

4. $\quad$ Sherman, D. A.; Murase, R.; Duyker, S. G.; Gu, Q.; Lewis, W.; Lu, T.; Liu, Y.; D'Alessandro, D. M., Reversible single crystal-tosingle crystal double [2+2] cycloaddition induces multifunctional photo-mechano-electrochemical properties in framework materials. Nat. Commun. 2020, 11, 2808.

5. Naumov, P.; Chizhik, S.; Panda, M. K.; Nath, N. K.; Boldyreva, E., Mechanically Responsive Molecular Crystals. Chem. Rev. 2015, 115, 12440-90.

6. Ahmed, E.; Karothu, D. P.; Naumov, P., Crystal Adaptronics: Mechanically Reconfigurable Elastic and Superelastic Molecular Crystals. Angew. Chem. Int. Ed. 2018, 57, 8837-8846. 
7. Commins, P.; Karothu, D. P.; Naumov, P., Is a Bent Crystal Still a Single Crystal? Angew. Chem. Int. Ed. 2019, 58, 10052-10060. 8. $\quad$ Gupta, P.; Karothu, D. P.; Ahmed, E.; Naumov, P.; Nath N. K., Thermally Twistable, Photobendable, Elastically Deformable, and Self-Healable Soft Crystals. Angew. Chem. Int. Ed. 2018, 57, 8498-8502.

9. $\quad$ Samanta, R.; Kitagawa, D.; Mondal, A.; Bhattacharya, M.; Annadhasan, M.; Mondal, S.; Chandrasekar, R.; Kobatake, S.; Reddy, C. M., Mechanical Actuation and Patterning of Rewritable Crystalline Monomer-Polymer Heterostructures via Topochemical Polymerization in a Dual-Responsive Photochromic Organic Material. ACS Appl. Mater. Interfaces 2020, 12, 16856-16863.

10. Seki, T.; Mashimo, T.; Ito, H., Anisotropic strain release in a thermosalient crystal: correlation between the microscopic orientation of molecular rearrangements and the macroscopic mechanical motion. Chem. Sci. 2019, 10, 4185-4191.

11. Seki, T.; Sakurada, K.; Muromoto, M.; Ito, H., Photoinduced single-crystal-to-single-crystal phase transition and photosalient effect of a gold(i) isocyanide complex with shortening of intermolecular aurophilic bonds. Chem. Sci. 2015, 6, 1491-1497.

12. Jin, M.; Yamamoto, S.; Seki, T.; Ito, H.; Garcia-Garibay, M. A., Anisotropic Thermal Expansion as the Source of Macroscopic and Molecular Scale Motion in Phosphorescent Amphidynamic Crystals. Angew. Chem. Int. Ed. 2019, 58, 18003-18010.

13. Samanta, R.; Ghosh, S.; Devarapalli, R.; Reddy, C. M., Visible Light Mediated Photopolymerization in Single Crystals: Photomechanical Bending and Thermomechanical Unbending. Chem. Mater. 2018, 30, 577-581.

14. Kitagawa, D.; Tsujioka, H.; Tong, F.; Dong, X.; Bardeen, C. J.; Kobatake, S., Control of Photomechanical Crystal Twisting by Illumination Direction. J. Am. Chem. Soc. 2018, 140, 4208-4212.

15. Hatano, E.; Morimoto, M.; Imai, T.; Hyodo, K.; Fujimoto, A.; Nishimura, R.; Sekine, A.; Yasuda, N.; Yokojima, S.; Nakamura S.; Uchida, K., Photosalient Phenomena that Mimic Impatiens Are Observed in Hollow Crystals of Diarylethene with a Perfluorocyclohexene Ring. Angew. Chem. Int. Ed. 2017, 56, 1257612580.

16. Zhu, L.; Al-Kaysi, R. O.; Bardeen, C. J., Photoinduced Ratchet-Like Rotational Motion of Branched Molecular Crystals. Angew. Chem. Int. Ed. 2016, 55, 7073-6.

17. Tong, F.; Xu, W.; Guo, T.; Lui, B. F.; Hayward, R. C.; Palffy-Muhoray, P.; Al-Kaysi, R. O.; Bardeen, C. J., Photomechanical molecular crystals and nanowire assemblies based on the [2+2] photodimerization of a phenylbutadiene derivative. J. Mater. Chem. C 2020, 8 (15), 5036-5044.

18. Mandal, R.; Garai, A.; Peli, S.; Datta, P. K.; Biradha, K., Photoinduced Bending of Single Crystals of a Linear Bis-Olefin via Water-Templated Solid-State [2+2] Photopolymerization Reaction. Chem. - Euro J. 2020, 26, 396-400.

19. Park, S. K.; Diao, Y., Martensitic transition in molecular crystals for dynamic functional materials. Chem. Soc. Rev. 2020, 49, 8287-8314.

20. Medishetty, R.; Husain, A.; Bai, Z.; Runcevski, T.; Dinnebier, R. E.; Naumov, P.; Vittal, J. J., Single crystals popping under UV light: a photosalient effect triggered by a [2+2] cycloaddition reaction. Angew. Chem. Int. Ed. 2014, 53, 5907-11.

21. Tong, F.; Kitagawa, D.; Dong, X.; Kobatake, S.; Bardeen, C. J., Photomechanical motion of diarylethene molecular crystal nanowires. Nanoscale 2018, 10, 3393-3398.

22. Irie, M.; Fukaminato, T.; Matsuda, K.; Kobatake, S., Photochromism of diarylethene molecules and crystals: memories, switches, and actuators. Chem. Rev. 2014, 114, 12174-277.

23. Rath, B. B.; Gallo, G.; Dinnebier, R. E.; Vittal, J. J., Reversible Thermosalience in a One-Dimensional Coordination Polymer Preceded by Anisotropic Thermal Expansion and the Shape Memory Effect. J. Am. Chem. Soc. 2021, 143, 2088-2096.
24. Tong, F.; Kitagawa, D.; Bushnak, I.; Al-Kaysi, R. O.; Bardeen, C. J., Light-Powered Autonomous Flagella-Like Motion of Molecular Crystal Microwires. Angew. Chem. Int. Ed. 2021, 60, 2414-2423.

25. Mahmoud Halabi, J.; Ahmed, E.; Sofela, S.; Naumov, P., Performance of molecular crystals in conversion of light to mechanical work. Proc. Natl. Acad. Sci. U. S. A. 2021, 118.

26. Naumov, P.; Sahoo, S. C.; Zakharov, B. A.; Boldyreva, E. V., Dynamic single crystals: kinematic analysis of photoinduced crystal jumping (the photosalient effect). Angew. Chem. Int. Ed. 2013, 52, 9990-5.

27. Das, S.; Mondal, A.; Reddy, C. M., Harnessing molecular rotations in plastic crystals: a holistic view for crystal engineering of adaptive soft materials. Chem. Soc. Rev. 2020, 49, 8878-8896.

28. Worthy, A.; Grosjean, A.; Pfrunder, M. C.; Xu, Y.; Yan, C.; Edwards, G.; Clegg, J. K.; McMurtrie, J. C., Atomic resolution of structural changes in elastic crystals of copper(II) acetylacetonate. Nat. Chem. 2018, 10, 65-69.

29. Panda, M. K.; Ghosh, S.; Yasuda, N.; Moriwaki, T.; Mukherjee, G. D.; Reddy, C. M.; Naumov, P., Spatially resolved analysis of short-range structure perturbations in a plastically bent molecular crystal. Nat. Chem. 2015, 7, 65-72.

30. Saha, S.; Desiraju, G. R., Crystal Engineering of HandTwisted Helical Crystals. J. Am. Chem. Soc. 2017, 139, 1975-1983.

31. Saha, S.; Mishra, M. K.; Reddy, C. M.; Desiraju, G. R., From Molecules to Interactions to Crystal Engineering: Mechanical Properties of Organic Solids. Acc. Chem. Res. 2018, 51, 2957-2967. 32. $\quad$ Ghosh, S.; Mishra, M. K.; Kadambi, S. B.; Ramamurty, U.; Desiraju, G. R., Designing elastic organic crystals: highly flexible polyhalogenated N-benzylideneanilines. Angew. Chem. Int. Ed. 2015, 54, 2674-8.

33. Seki, T.; Feng, C.; Kashiyama, K.; Sakamoto, S.; Takasaki, Y.; Sasaki, T.; Takamizawa, S.; Ito, H., Photoluminescent Ferroelastic Molecular Crystals. Angew. Chem. Int. Ed. 2020, 59, 8839-8843.

34. Reddy, C. M.; Gundakaram, R. C.; Basavoju, S.; Kirchner, M. T.; Padmanabhan, K. A.; Desiraju, G. R., Structural basis for bending of organic crystals. Chem. Commun. 2005, 3945-3947.

$35 . \quad$ Rath, B. B.; Vittal, J. J., Single-Crystal-to-Single-Crystal [2 + 2] Photocycloaddition Reaction in a Photosalient OneDimensional Coordination Polymer of Pb(II). J. Am. Chem. Soc. 2020, 142, 20117-20123.

36. Al-Kaysi, R. O.; Müller, A. M.; Bardeen, C. J., Photochemically driven shape changes of crystalline organic nanorods. J. Am. Chem. Soc. 2006, 128, 15938-15939.

37. Kobatake, S.; Takami, S.; Muto, H.; Ishikawa, T.; Irie, M., Rapid and reversible shape changes of molecular crystals on photoirradiation. Nature 2007, 446, 778-781.

38. $\quad$ Mei, L.; An, S. W.; Hu, K. Q.; Wang, L.; Yu, J. P.; Huang, Z. W.; Kong, X. H.; Xia, C. Q.; Chai, Z. F.; Shi, W. Q., Molecular Springlike Triple-Helix Coordination Polymers as Dual-Stress and Thermally Responsive Crystalline Metal-Organic Materials. Angew. Chem. Int. Ed. 2020, 59, 16061-16068.

39. Dakovic, M.; Borovina, M.; Pisacic, M.; Aakeroy, C. B.; Soldin, Z.; Kukovec, B. M.; Kodrin, I., Mechanically Responsive Crystalline Coordination Polymers with Controllable Elasticity. Angew. Chem. Int. Ed. 2018, 57, 14801-14805.

40. Bhattacharya, B.; Michalchuk, A. A. L.; Silbernagl, D.; Rautenberg, M.; Schmid, T.; Feiler, T.; Reimann, K.; Ghalgaoui, A.; Sturm, H.; Paulus, B.; Emmerling, F., A Mechanistic Perspective on Plastically Flexible Coordination Polymers. Angew. Chem. Int. Ed. 2020, 59, 5557-5561.

41. Dutta, B.; Sinha, C.; Mir, M. H., The sunlight-driven photosalient effect of a 1D coordination polymer and the release of an elusive cyclobutane derivative. Chem. Commun. 2019, 55, 11049-11051. 
42. Shi, Y. X.; Zhang, W. H.; Abrahams, B. F.; Braunstein, P.; Lang, J. P., Fabrication of Photoactuators: Macroscopic Photomechanical Responses of Metal-Organic Frameworks to Irradiation by UV Light. Angew. Chem. Int. Ed. 2019, 58, 9453-9458. 43. Wang, H.; Chen, P.; Wu, Z.; Zhao, J.; Sun, J.; Lu, R., Bending, Curling, Rolling, and Salient Behavior of Molecular Crystals Driven by [2+2] Cycloaddition of a Styrylbenzoxazole Derivative. Angew. Chem. Int. Ed. 2017, 56, 9463-9467.

44. Li, S.; Yan, D., Tuning Light-Driven Motion and Bending in Macroscale-Flexible Molecular Crystals Based on a Cocrystal Approach. ACS Appl. Mater. Interfaces 2018, 10, 22703-22710.

45. Schmidt, G. M. J., Photodimerization in the solid state. Pure Appl. Chem. 1971, 27, 647-678.

46. Rath, B. B.; Kole, G. K.; Vittal, J. J., Structural Transformation of Photoreactive Helical Coordination Polymers to Two-Dimensional Structures. Cryst. Growth Des. 2018, 18, 62216226.

47. Rath, B. B.; Kole, G. K.; Morris, S. A.; Vittal, J. J., Rotation of a helical coordination polymer by mechanical grinding. Chem. Commun. 2020, 56, 6289-6292.

48. Medishetty, R.; Sahoo, S. C.; Mulijanto, C. E.; Naumov, P.; Vittal, J. J., Photosalient Behavior of Photoreactive Crystals. Chem. Mater. 2015, 27, 1821-1829.

49. Kim, T.; Al-Muhanna, M. K.; Al-Suwaidan, S. D.; Al-Kaysi, R. O.; Bardeen, C. J., Photoinduced curling of organic molecular crystal nanowires. Angew. Chem. Int. Ed. 2013, 52, 6889-93.

50. Al-Kaysi, R. O.; Tong, F.; Al-Haidar, M.; Zhu, L.; Bardeen, C. J., Highly branched photomechanical crystals. Chem. Commun. 2017, 53, 2622-2625. 


\section{For Table of Contents Use Only}

Title: Mechanical Bending and Modulation of Photoactuation Properties in a One-Dimensional Pb(II) Coordination Polymer

Authors: Bibhuti Bhusan Rath, and Jagadese J. Vittal*

Table of Contents Graphics

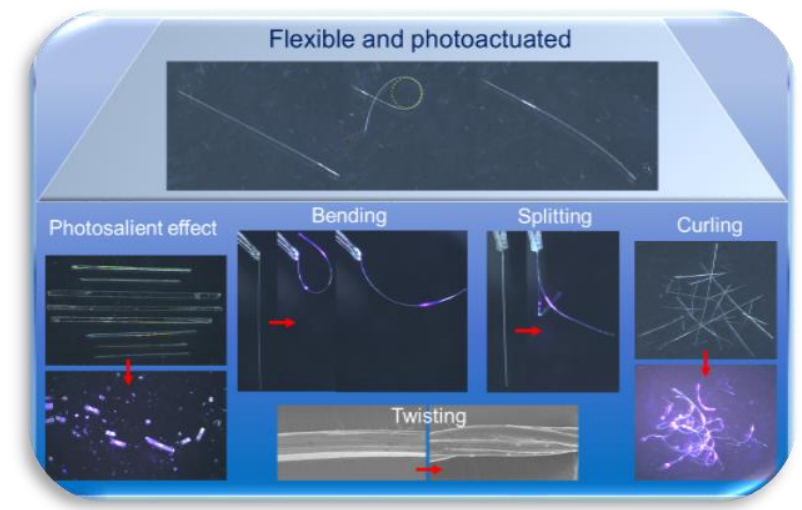

